\title{
Prevalence of premenstrual syndrome and associated factors among Debrebirhan Town, North Showa, Amhara Region, Ethiopia
}

\author{
Desalegn Asmare ${ }^{1}$, Gezahegn Nekatibeb ${ }^{2}$, Zegeye Merhatibeb ${ }^{3}$, Meseret Gizachew ${ }^{4}$ \\ ${ }^{1}$ Department of Psychiatry, Debrebirhan Referral Hospital, Ethiopia \\ ${ }^{2}$ Department of Paediatrics and Child Health, Debrebirhan Referral Hospital, Ethiopia \\ ${ }^{3}$ Departement Outpatient Clinic, Debrebirhan Referral Hospital, Ethiopia \\ ${ }^{4}$ Departement of ICT, Debrebirhan Town ICT officer, Ethiopia

\section{Email address:} \\ asmaredesalegn@gmail.com (D. Asmare),drgezahegn@yahoo.com (G. Nekatibeb), merha1968@gmail.com (Z. Merhatibeb), \\ meseretgizachew23@gmail.com (M. Gizachew)
}

\section{To cite this article:}

Desalegn Asmare, Gezahegn Nekatibeb, Zegeye Merhatibeb, Meseret Gizachew. Prevalence of Premenstrual Syndrome and Associated Factors among Debrebirhan Town, North Showa, Amhara Region, Ethiopia. American Journal of Psychiatry and Neuroscience.

Vol. 3, No. 1, 2015, pp. 8-14. doi: 10.11648/j.ajpn.20150301.12

\begin{abstract}
PMS or premenstrual syndrome is a condition that manifests as emotional, physical and behavioral symptoms and affects women between their late 20's to early 40's. The condition is marked by the symptoms 5 to 10 days prior to beginning of the period and symptoms resolve once the period begins or within 4 to 7 days. PMS is a relatively common condition and almost $75 \%$ women suffer from this condition at some point in their lives. Sometimes mood swings, behavioural changes and physical symptoms may be severe enough to affect normal life. Objectives: The aim of this study is to assess the prevalence of premenstrual syndrome and associated factors among Debrebirhan Town, North Showa, and Amhara Region, Ethiopia. Methods: a community based cross-sectional quantitative study was conducted among Debrebirhan residence. A pre-tested structured and semi structured face to face interview questionnaire was used. Data was collected from September to November, 2013 after obtaining verbal consent from the respondent. Data entry was performed by using SPPSS version 20 and analysis using logistic regression and odd ratio. Results: A total of 321 participants $41.12 \%$ were premenstrual syndrome. Premenstrual syndrome was associated with Income less than 400 birr $(A O R=2.766(1.290,5.927))$ is associated with PMS. Those with income less than 400birrs are 2.766times more likely to PMS than those who have income greater than 700birr. Income between 400-700birr $(\mathrm{AOR}=2.684(1.155,6.236)$ ) is associated with PMS. Those with income between 400-700birr are 2.684times more likely to PMS than their have income greater than 700birr. Previous history of depression is $(\mathrm{AOR}=1.843(1.110,3.059))$ is associated with depression. Those with Previous history of depression are 1.843 times likely to develop PMS than those who have not previous history of depression. Continuous follow up in hospital (AOR=13.915(1.471, 131.674)) is associated with PMS, those with Continuous follow up in hospital are 13.915times likely to develop PMS than those who have not continuous follow up in hospital. Conclusion and recommendation: The aim of this study was to estimate the prevalence of premenstrual syndrome among Debrebirhan residence to assess the factors that are associated with PMS. From the findings of this study, it emerged that PMS is high among who participated in the study. For all the sociodemographic factors and related factors that were studied, it emerged from the findings of the study. That continuous follow up in hospital, previous history of depression and low income were statistically associated with scoring Positive to PMS.
\end{abstract}

Keywords: Premenstrual Syndrome, Premenstrual Dysphoric Disorder, Debrebirhan Town

\section{Introduction}

Premenstrual dysphoric disorder (PMDD) is a mood disorder with onset of functionally impairing or distressing mood symptoms in the late luteal phase of the menstrual cycle. Psycho physiologic findings in PMDD broadly fall into two categories: vulnerability trait findings, thus categorized because they are present in the asymptomatic phases of the menstrual cycle, and state findings, which are 
only present in the symptomatic late luteal phase and which are potentially representative of the hormonal events and biological mechanisms that lead to PMDD. Trait vulnerability markers in PMDD include diminished cardiovascular stress responses, lower heart rate variability (reflecting increased vagal tone), and lower P300 amplitude, eventually suggesting that women with PMDD share a number of physiological correlates with related anxiety and mood disorders. State findings in PMDD include lower luteal phase prepulse inhibition and altered luteal phase emotion processing. Lower prepulse inhibition in the late luteal phase may be an important ovarian steroid-influenced indicative of altered serotonergic neurotransmission, of relevance for women with PMDD. Attempts to determine the neural correlates of emotion processing in the late luteal phase are thus far inconsistent, but promising(1).

Study done at the Harvard the diagnosis of PMDD was confirmed in 33 of 513 women $(6.4 \%)$ who completed the prospective evaluation with daily records. Fourteen subjects $(2.7 \%)$ met criteria for PMDD without a previous history of depression. PMDD was associated with lower education (odds ratio $[\mathrm{OR}]=2.3$, confidence interval $[\mathrm{CI}]=1.1-4.9$ ), a history of major depression $(\mathrm{OR}=3.6, \mathrm{CI}=1.7-7.4)$, and current cigarette smoking $(\mathrm{OR}=4.1, \mathrm{CI}=1.5-11.1)$. In addition, women not working outside the home were significantly less likely to meet criteria for PMDD $(\mathrm{OR}=0.2, \mathrm{CI}=0.1-0.9)(2)$.

The prevalence of PMDD is $13-18 \%$ of women of reproductive age may have premenstrual dysphoric symptoms severe enough to induce impairment and distress, though the number of symptoms may not meet the arbitrary count of 5 symptoms on the PMDD list..Appropriate recognition of the disorder and its impact should lead to treatment of more women with PMS/PMDD(3).The baseline 12-month prevalence of DSM-IV PMDD was 5.8\%(4).

The incidence of threshold PMDD was $3.0 \%$. The most powerful predictors were sub threshold PMDD at baseline $(\mathrm{OR}=11.0,95 \% \mathrm{CI}=4.7$ to 25.9$)$. Traumatic events greatly increased the odds of developing PMDD at follow-up (OR = $4.2,95 \% \mathrm{CI}=1.2$ to 12.0$)$. Other predictors were a history of anxiety disorder ( $\mathrm{OR}=2.5,95 \% \mathrm{CI}=1.1$ to 5.5$)$ and elevated daily hassles scores $(\mathrm{OR}=1.6,95 \% \mathrm{CI}=1.1$ to 2.3$)$. Both were also associated with the risk of developing sub threshold PMDD, although the association was less robust(5).

A total of 3,913 women aged 15 to 54 years answered the questions on PMS symptoms, and 3,522 of them additionally answered the questions on interference of PMS with life. Ninety one percent of the participants reported at least one symptom, $10.3 \%$ had PMS and $3.1 \%$ fulfilled the criteria for PMDD. The prevalence of PMS was higher in nonmarried women, in women aged 35-44 years and in women of the Italian-speaking region of Switzerland. Both PMS and PMDD were strongly associated with poor physical health and psychological distress. Considering the association with poor physical health and high psychological distress, a broader underlying vulnerability in women qualifying for PMDD must be assumed and should be taken into account in clinical management as well as in future research in this field(6).

In a longitudinal study of their health and development, 384 15-year-old females reported their experience of symptoms indicative of premenstrual syndrome (PMS). The prevalence of these symptoms is reported and a group of adolescents is identified with the syndrome (14\%). PMS was associated with current self-reported anxiety, inattention, and poor health. Preadolescent self-report and maternal ratings of physical and mental health did not significantly predict adolescent PMS. The results suggest that the experience of PMS in adolescence may be mediated by perceived health status; the roles of mental health and maternal influence in the development of adolescent PMS may be minimal(7).

Study done in Iranian of the 300 participants, 98.2\% reported at least one mild to severe premenstrual symptom and $16 \%$ met the criteria of DSM-IV for PMS. Most common symptoms were feeling of tiredness or lethargy (84\%), depressed mood $(72.3 \%)$, sudden feeling of sadness or tearfulness $(70.3 \%)$, anxiety $(70 \%)$, backache $(69 \%)$ and sleep problems $(66 \%)(8)$.

One hundred and five adolescent girls out of $171(61.4 \%)$ met DSM-IV criteria for PMS. There was an association between dysmenorrhea and PMS in $60(57.1 \%)$. Half of the girls, i.e. 52 (49.5\%) had mild, 39 (37.1\%) had moderate and $14(13.4 \%)$ had severe PMS. The most common symptom of PMS was negative affect particularly in the form of stress $(87.6 \%)$ and nervousness $(87.6 \%)$. There was a statistically significant negative relationship between milk consumption and the following: abdominal bloating, cramps, craving for some foods and increased appetite.Conclusion: PMS and dysmenorrhea is frequently overlapping. We also found that PMS is associated with dietary habits(9).

In United Arab Emirates the prevalence of premenstrual syndrome was $16.4 \% \quad(n=115)$. On logistic regression analysis, premenstrual syndrome was significantly associated with Emirati nationality ( $\mathrm{p}=0.001)$, presence of the condition in sister $(\mathrm{s})(\mathrm{p}=0.002)$, and dysmenorrhea $(\mathrm{p}<0.001)$. Only 52 affected subjects $(45.2 \%)$ were currently taking treatment for premenstrual syndrome and the majority (60\%) used pharmacological therapy. Premenstrual syndrome had a moderate but significant negative impact $(\mathrm{p}<0.001)$ on the quality of life of affected girls, particularly school performance, social interactions, lifestyle, and emotional well-being. Difficulty in performing school functions and decrease in stigma were the two most adversely affected parameters (10).

Study done at Thai Nurses the prevalence of PMS in was $25.1 \%$. Nurses with younger age, lower income, more coffee consumption, dysmenorrheal, and negative attitude toward menstruation had higher prevalence of PMS. After multiple logistic regression analysis, the significant factors associated with PMS were coffee consumption $>1$ cups/day and negative attitude toward menstruation; odds ratios $(95 \%$ confidence interval) were 2.322 (1.257 to 4.288$)$ and 5.768 (2.096 to 15.872), respectively(11).

Study done in polish the mean age of the studied population was $17.27 \pm 0.97$ years. In the studied population 
the majority of the adolescent girls lived in large cities of over 50000 citizens $(54.29 \%)$, were physically active declaring daily physical exercise $(55.22 \%)$ and sexually active $(54.17 \%)$. Study results indicated that the prevalence of premenstrual syndrome and premenstrual dysphoric disorder was $76.39 \%$ and $4.17 \%$, respectively. The final statistical analysis revealed that only place of residence (large cities) increased the risk of premenstrual syndrome $(\mathrm{OR}=3.58 ; \mathrm{P}=0.01)(12)$.

In Jimma University the age of participants ranged from 17 to 38 years, with mean \& median age of $20.3 \& 20$ years, respectively. Almost all (99.6\%) had at least one premenstrual (PM) symptom in many of the menstrual cycles in the last 12 months. The prevalence of PMS or premenstrual dysphoric disorder (according to DSM-IV) was $27 \%$. About $14 \%$ of the study participants frequently missed classes and $15 \%$ missed examinations or scored a lower grade at least once because of PM symptoms(13).

In United Kingdom Southampton Of the women surveyed, $24 \%$ were considered to have premenstrual symptoms $(95 \%$ confidence interval [CI] 21-27). Women were less likely to have symptoms if they had higher levels of educational attainment and suffered less from stress. No associations were found between premenstrual symptoms and diet, alcohol, or strenuous exercise or after adjustment for other factors, with age, smoking, or body mass index (BMI)(14).

It was determined that the participants' mean age was $20.56 \pm 1.77$. Approximately half of the girls (49.7\%) had PMS. According to the PMSS subscale scores, 205 girls (68.3\%) had changes in appetite, $197(65.7 \%)$ had irritability, $196(65.3 \%)$ had swelling, $143(47.7 \%)$ had fatigue, 185 (61.7\%) had pain, 154 (51.3\%) had depressive feelings, 140 (46.7\%) had changes in sleeping habits, 102 (34.0\%) had depressive thoughts, and $56(18.7 \%)$ had anxiety. It was determined that PMS mean scores increased in parallel with problems that affect the mood of the girls. The contributing factors for PMS in the girls were found to be having premenstrual and menstrual pain (P 0.036), having mothers with PMS $(\mathrm{P}=0.045)$, and having irregular menstruations after coming to university $(\mathrm{P}=0.000)(15)$.

Premenstrual syndrome (PMS) has been studied in many countries, but few studies have been reported internationally on the experience of Chinese women. Because culture and employment are important factors in the perception of health status, in this study we sought to determine the existence and features of PMS in Chinese clerical women in Hong Kong. We used a cross-sectional, retrospective approach to collect data with a translation of an established questionnaire (the Menstrual Distress Questionnaire). Fatigue was found to be the most prevalent symptom, and the Pain, Water Retention, Behavioral Change, and Negative Affect scales had more than $64 \%$ frequency. The main difference between these findings and those of other studies is that negative affect featured most prominently in Western samples, whereas pain featured most highly in this sample of Chinese women(16).

Overall 1,477 women started the study-of these, 822 (56\%) completed the study as planned and represent the full analysis set. Employed women with moderate-to-severe PMS/PMDD had higher rate of productivity impairment on the modified version of the WPAI questionnaire (values $\geq 7$ ) relative to those with no perceived symptoms/mild PMS (adjusted odds ratio, 3.12; 95\% confidence interval, 1.755.57). Similar outcomes were obtained for impairment of working productivity or efficiency using the PSST scale (value 4). The mean number of days on the DRSP with at least moderate reduction in productivity or efficiency in daily routine was higher for women with moderate-to-severe PMS/PMDD (5.6 vs. 1.1). Women with moderate-to-severe PMS/PMDD had a higher rate of absenteeism ( $>8$ hours per cycle; $14.2 \%$ vs. $6.0 \%(17)$ ).

In Rizwana Durrani the frequency of premenstrual syndrome was $53 \%$ according to ICD-10 criteria, among which $42 \%$ was mild, $18.2 \%$ moderate and $31.7 \%$ severe. A total of 64 girls (18.2\%) met the DSM-IV criteria for severe PMS or Premenstrual Dysphoric disorder (PMDD). The order of frequency of symptoms occurring in PMS was general body discomfort, anxiety, backache, fatigue and depression. Most frequently reported symptoms in PMDD group were anger, anxiety, stress, depression, fatigue and general body discomfort(18)

Premenstrual dysphoric disorder (PMDD) represents the more severe and disabling end of the spectrum of premenstrual syndrome and occurs in an estimated $2 \%$ to $9 \%$ of menstruating women. The most frequent PMDD symptoms among women seeking treatment consist of anger/irritability, anxiety/tension, feeling tired or lethargic, mood swings, feeling sad or depressed, and increased interpersonal conflicts. Women who develop PMDD appear to have serotonergic dysregulation that may be triggered by cyclic changes in gonadal steroids. The marked increase in the number of well-designed placebo-controlled studies in the past decade has established several selective serotonin reuptake- inhibiting antidepressants as effective first-line treatments for this disorder(19).

The premenstrual syndrome (PMS) is a major clinical entity afflicting a large segment of the female population. Available information is descriptive in nature and the etiology of this syndrome remains unclear. In this review, both biochemical and psychosocial elements of the syndrome have been explored in an effort to redefine the pathophysiology of this seemingly multifactorial psychoneuroendocrine dysfunction. We propose that luteal phase sensitivity to and subsequent withdrawal from the central effects of the neuropeptides beta-endorphin and alpha-melanocyte-stimulating hormone result in a cascade of neuro endocrine changes within the brain-hypothalamuspituitary complex. Modulation of neurotransmitter function by these peptides may produce alterations in mood and behavior as well as enhance pituitary release of prolactin and vasopressin. Variable gonadal steroid modulation of these responses from subject to subject likely accounts for the heterogeneous clinical manifestations of the $\operatorname{PMS}(20)$.

Premenstrual syndrome, a common cyclic disorder of 
young and middle-aged women, is characterized by emotional and physical symptoms that consistently occur during the luteal phase of the menstrual cycle. Women with more severe affective symptoms are classified as having premenstrual dysphoric disorder. are diagnoses of exclusion; therefore, alternative explanations for symptoms must be considered before either diagnosis is made. The disorders can manifest with a wide variety of symptoms, including depression, mood lability, abdominal pain, breast tenderness, headache, and fatigue. Women with mild symptoms should be instructed about lifestyle changes, including healthy diet, sodium and caffeine restriction, exercise, and stress reduction. Supportive strategies, such as use of a symptom diary, may be helpful in diagnosing and managing the disorders. In women with moderate symptoms, treatment includes both medication and lifestyle modifications(21).

\subsection{Justification of the Study}

The World Health Organization report on PMS and mental illness has indicated that awareness about premenstrual syndrome/premstrual dysphoric disorder very low. The findings of this study would add to the limited body of knowledge about Prevalence of premenstrual dysphoric disorder and associated factors among in Ethiopia. This will serve as a clinical reference to PMS care providers who may use the findings of this study.

Since little is known about Prevalence of premenstrual syndrome and associated factors among in Ethiopia, this study might serve as a foundation for future public health and Mental Health research in the area, thus deepening Understanding of the issue. It is therefore anticipated that the study findings will contribute to the development of local knowledge about PMS and be used to inform Mental Health education curricula for both community and mental Health.

The findings of this study might also help in influencing the development of appropriate policies, plans and intervention programs for the management and Treatment of psychiatric illnesses in PMS/PMDD treatment settings.

\section{Objective}

\subsection{General Objective}

- prevalence of premenstrual syndrome and associated factors among Debrebirhan residence, North Showa, Amhara Region, Ethiopia.2013

\subsection{Specific Objectives}

- To determine the prevalence of premenstrual syndrome among in Debrebirhan residence, North Showa, Amhara Region, Ethiopia, 2013.

- To identify the associated factors of premenstrual syndrome among in Debrebirhan residence, North Showa, Amhara Region, Ethiopia, 2013.

\section{Methods}

\subsection{Study Design}

- Community based cross-sectional study design was employed.

\subsection{Study Area}

The study was conducted in Debrebirhan residence, Debrebirhan town, and North Showa Ethiopia from September to November, 2013. Debrebirhan Town located in the North Showa Zone of Amhara Regional State located, $695 \mathrm{~km}$ from the Bahirdar and $130 \mathrm{~km}$ from the capital of Ethiopia, Addis Ababa. Administratively, Debrebirhan District has 9 urban kebeles (smallest administrative units) with a projected. Based on the 2007 Census conducted by the Central Statistical Agency of Ethiopia (CSA), Debrebirhan Town has an estimated total population of 61,025.In the study area, there are one Referral Hospital, one private Hospital, three health centre and five private clinics and six private pharmacies.

\subsection{Study Period}

The study was conducted from September to November, 2013.

\subsection{Source Population}

Source population was comprised of all people living in at Debrebirhan residence.

\subsection{Study Population}

Study population is comprised of all people living kebeles 1,8 , and 5 at Debrebirhan residence.

\subsection{Data Collection Instrument}

A structured questionnaire was used to collect the data on socio-demographic characteristics (age, ethnicity, religion, education, occupation, and marital status). PSST (premenstrual syndrome screening tool) was used to measure the PMS. A format or questionnaire format was used substance use, previous history of depression, continuous follow-up at hospital. The researcher was recruiting two nurses to collect the data. They were oriented on the how to use of the questionnaire and the ethical principles of confidentiality and data management prior to their involvement with data collection and data will be collected for thirty days.

\subsection{Data Quality Control Issues}

Data quality control issues were insured by conducting pre-test among 5\% total samples obtained from patients attending at kebeles-9. Training was given to the data collectors on the data collection tool and sampling techniques Supervision was held regularly during data collection period. The collected data was checked on daily basis for completeness and consistence. The questionnaire is translated 
to Amharic and back to English backward forward translation method was used and feedback was acquired from two psychiatrist who have worked on depression most of their lives.

\subsection{Data Processing and Analysis}

The coded data was checked, cleaned by entering into Epi.Info version 7 and then exported into Statistical Package for the Social Sciences (SPSS window version 20) for analysis, descriptive summary using frequencies, proportions, graphs and cross tabs used to present study results. Bivariate analysis performed to determine each of these factors and how they are associated with dependant variable. Only factors that have been found to be significantly associated with PMS during bivariate analysis were entered into the multivariate analysis. $\mathrm{P}$ value $<0.05$ was statistically significant.

\subsection{Ethical Considerations}

Ethical clearance was obtained from Debrebirhan referral hospital. Moreover the researcher was striving to protect and to respect the privacy, secrecy and wellbeing of persons with these conditions. The data that was collected for the purpose of this study was never containing identifying information, thus ensuring the secrecy of the participants. All the data to be collected was used for the purpose of this study only. Verbal consent to participate was obtained from participants and an outline of the purpose of the study discussed with each client who agreed to voluntary participation.

\section{Result}

Prevalence of premenstrual syndrome among Debrebirhan town, North Showa, Amhara Region, Ethiopia.2014

The prevalence of PMS was assessed using PSST. The prevalence of PMS was found to be $41.12 \%$.

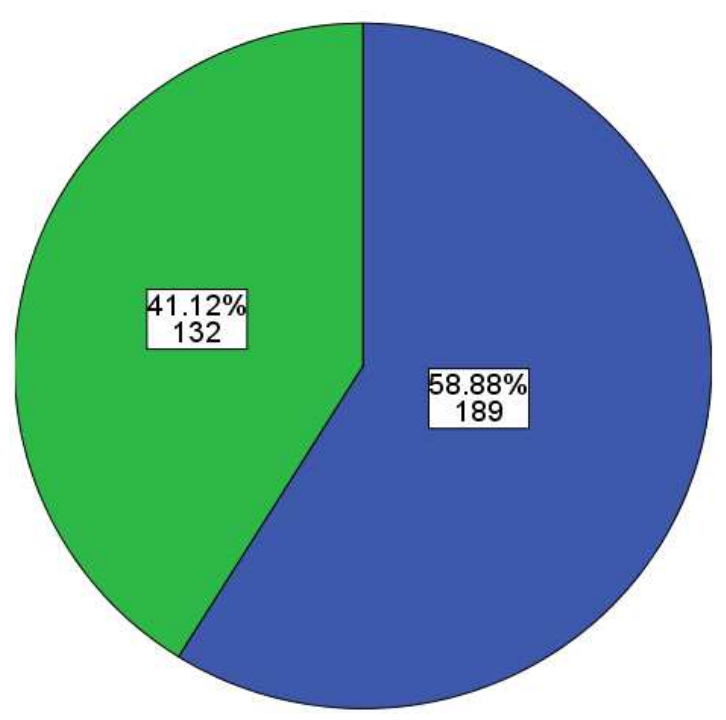

Figure 1. Prevalence of premenstrual syndrome and associated factors among Debrebirhan town, North Showa, Amhara Region, Ethiopia.2014
Table 1. Distribution sociodemographic variable Prevalence of premenstrual syndrome and associated factors among Debrebirhan town, North Showa, Amhara Region, Ethiopia.

\begin{tabular}{|c|c|c|c|}
\hline \multicolumn{2}{|c|}{ Sociodemographic variables } & \multirow{2}{*}{$\begin{array}{l}\text { Frequency } \\
85\end{array}$} & \multirow{2}{*}{$\begin{array}{l}\text { Percent (\%) } \\
26.5\end{array}$} \\
\hline & 15-24year & & \\
\hline Age category & 25-34year & 171 & 53.3 \\
\hline & 35-44year & 65 & 20.2 \\
\hline \multirow{3}{*}{ Ethnicity } & Amhara & 299 & 93.1 \\
\hline & Oromo & 17 & 5.3 \\
\hline & Tigre & 5 & 1.6 \\
\hline \multirow{4}{*}{ Marital status } & single & 71 & 22.1 \\
\hline & married & 145 & 45.2 \\
\hline & divorced & 56 & 17.4 \\
\hline & windowed & 49 & 15.3 \\
\hline \multirow{3}{*}{ Religion } & orthodox & 296 & 92.2 \\
\hline & Muslim & 13 & 4.0 \\
\hline & Protestant & 12 & 3.7 \\
\hline \multirow{5}{*}{ Education } & illiterate & 59 & 18.4 \\
\hline & $\begin{array}{l}\text { Able to write and } \\
\text { read }\end{array}$ & 36 & 11.2 \\
\hline & primary & 117 & 36.4 \\
\hline & secondary & 81 & 25.2 \\
\hline & tertiary & 28 & 8.7 \\
\hline \multirow{3}{*}{ Income } & $<400$ birr & 169 & 52.6 \\
\hline & 400birr-700birr & 80 & 24.9 \\
\hline & $>700$ birr & 72 & 22.4 \\
\hline \multirow{2}{*}{ Jobs } & employment & 238 & 74.1 \\
\hline & unemployment & 83 & 25.9 \\
\hline \multirow{2}{*}{$\begin{array}{l}\text { Continuous } \\
\text { follow up in } \\
\text { hospital }\end{array}$} & no & 316 & 98.4 \\
\hline & yes & 5 & 1.6 \\
\hline \multirow{2}{*}{$\begin{array}{l}\text { Previous history } \\
\text { of depression }\end{array}$} & no & 218 & 67.9 \\
\hline & yes & 103 & 32.1 \\
\hline \multirow{2}{*}{ Alcohol user } & no & 269 & 83.8 \\
\hline & yes & 52 & 16.2 \\
\hline \multirow{2}{*}{$\begin{array}{l}\text { More consumption } \\
\text { of coffee }\end{array}$} & no & 92 & 67 \\
\hline & yes & 97 & 65 \\
\hline \multirow{2}{*}{$\begin{array}{l}\text { Previous history } \\
\text { of migraine } \\
\text { headache }\end{array}$} & no & 144 & 45 \\
\hline & yes & 93 & 39 \\
\hline
\end{tabular}

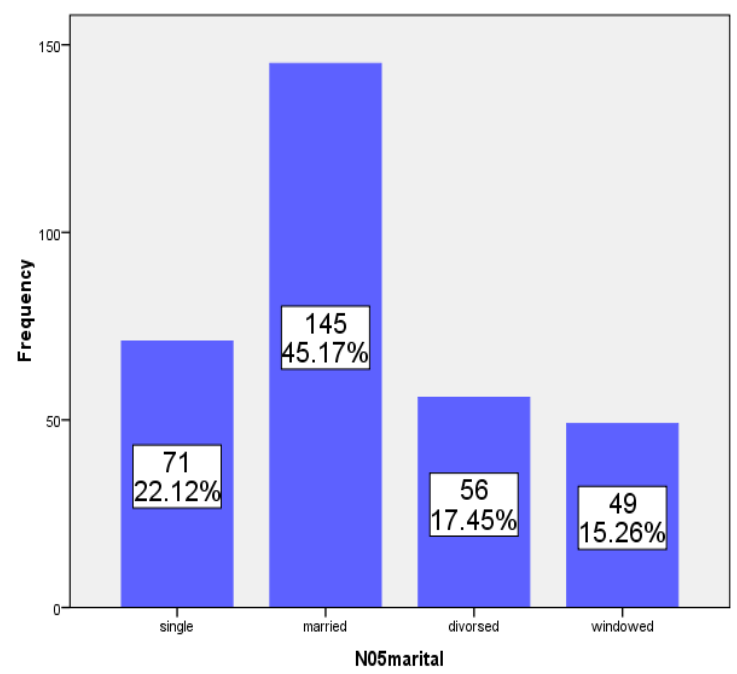

Figure 2. distribution of premenstrual syndrome by sex among the study participants, Debrebirhan town, Amhara Region, Ethiopia. 
Income less than 400 birr $(\mathrm{AOR}=2.766(1.290,5.927))$ is associated with PMS. Those with income less than 400birrs are 2.766times more likely to PMS than those who have income greater than 700birr. Income between 400-700birr $(\mathrm{AOR}=2.684(1.155,6.236))$ is associated with PMS. Those with income between 400-700birr are 2.684times more likely to PMS than their have income greater than 700birr.

Previous history of depression is $(\mathrm{AOR}=1.843(1.110$, 3.059)) is associated with depression. Those with Previous history of depression are 1.843times likely to develop PMS than those who have not previous history of depression.

Continuous follow up in hospital $(\mathrm{AOR}=13.915(1.471$, 131.674)) is associated with PMS, those with Continuous follow up in hospital are 13.915times likely to develop PMS than those who have not continuous follow up in hospital.

No association is observed between PMS and age, ethnicity, marital status, religion, jobs, substance use. But educational status are associated in bivariate but not associated in multivariate

Table 2. bivariate analysis and multivariate analysis of factors and PMS among the study participant, Debrebirhan town, North Showa, Amhara Region, Ethiopia, 2013.

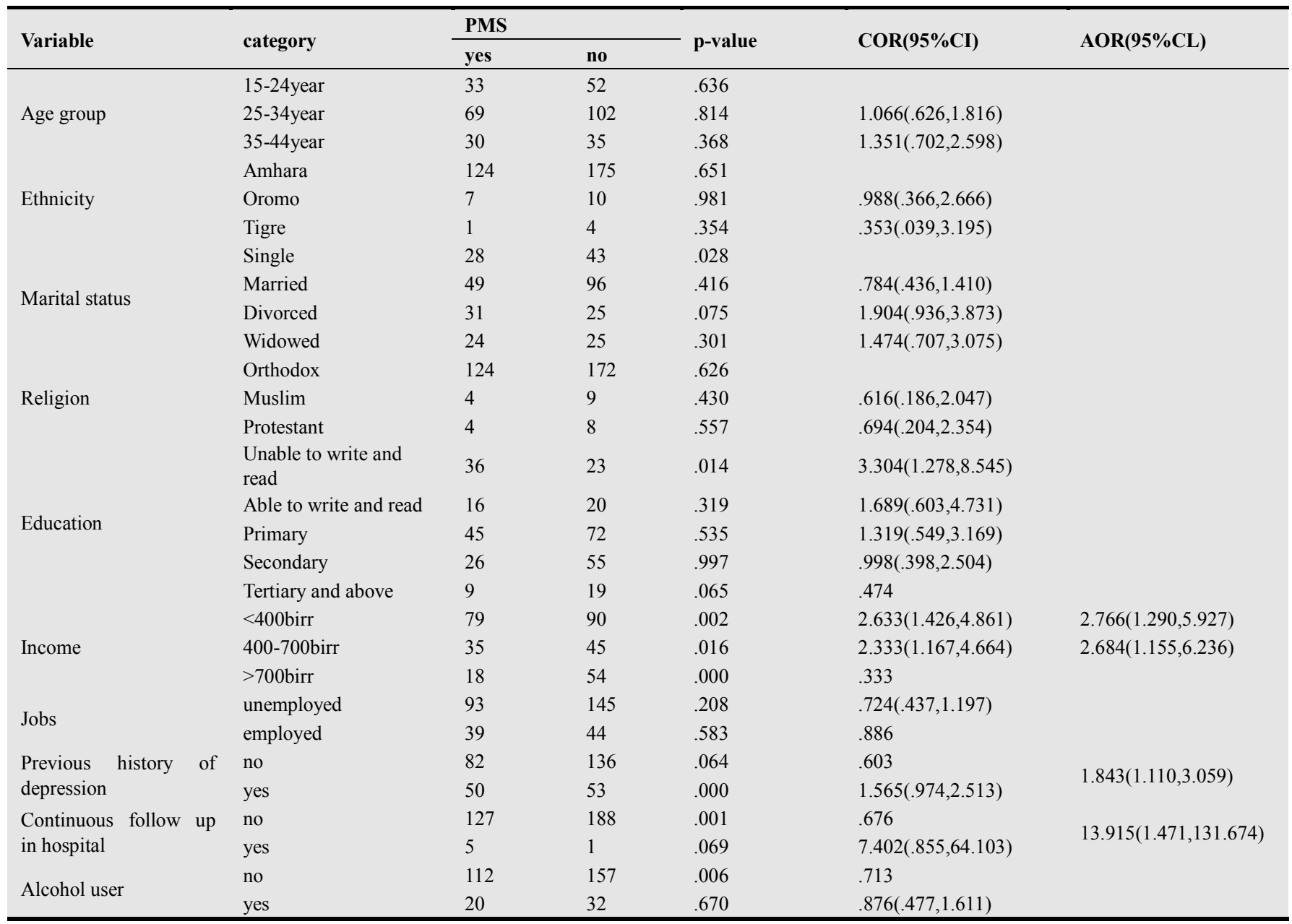

\section{Discussion}

In this study the prevalence of premenstrual syndrome is $41.12 \%$. This cross-sectional study found that the significant associations of where: previous history of depression, continuous follows up in hospital, income is significantly associated with PMS in the multivariable analysis. On the other hand educational status being unable to write and read is significant association in bivariate analysis but not significant association with multivariate analysis.

The study showed that there is a high prevalence of premenstrual syndrome among Debrebirhan residence when compared to the general population. Across-sectional study conducted in Iranian Of the 300 participants $16 \%$ met the criteria of DSM-IV for PMS .this result is higher than when compared with this research(8).

The prevalence rate of premenstrual syndrome was $41.12 \%$ which is higher than a study done at Thai Nurses, Thai in which the prevalence is reported to be $25.1 \%(11)$.

The prevalence was higher than study done in United Kingdom Southampton Of the women surveyed, 24\% were considered to have premenstrual symptoms (95\% confidence interval, the possible reason might be the difference of instruments used to assess premenstrual syndrome, this might also be due to the different study design and sample size 
used(14).

The result of the study is also lower than the study conducted in Rizwana Durrani the frequency of premenstrual syndrome was $53 \%$ according to ICD-10 criteria(18).

This result is lower and higher when compared to the result reported with other study. The possible reason might be the difference of instruments used to assess depression this might also be due to the different study design and sample size used.

\section{Conclusion}

The aim of this study was to estimate the prevalence of premenstrual syndrome among Debrebirhan residence to assess the factors that are associated with PMS. From the findings of this study, it emerged that PMS is high among who participated in the study. For all the socio-demographic factors and related factors that were studied, it emerged from the findings of the study. That continuous follow up in hospital, previous history of depression and low income were statistically associated with scoring Positive to PMS.

\section{References}

[1] Poromaa IS. Physiological Correlates of Premenstrual Dysphoric Disorder (PMDD). Current topics in behavioral neurosciences. 2014;21:229-43.

[2] Cohen LS, Soares CN, Otto MW, Sweeney BH, Liberman RF, Harlow BL. Prevalence and predictors of premenstrual dysphoric disorder (PMDD) in older premenopausal women: the Harvard Study of Moods and Cycles. Journal of affective disorders. 2002;70(2):125-32.

[3] Halbreich U, Borenstein J, Pearlstein T, Kahn LS. The prevalence, impairment, impact, and burden of premenstrual dysphoric disorder (PMS/PMDD). Psychoneuroendocrinology. 2003;28:1-23.

[4] Wittchen H-U, Becker E, Lieb R, Krause P. Prevalence, incidence and stability of premenstrual dysphoric disorder in the community. Psychological medicine. 2002;32(01):119-32.

[5] Perkonigg A, Yonkers KA, Pfister H, Lieb R, Wittchen H-U. Risk factors for premenstrual dysphoric disorder in a community sample of young women: the role of traumatic events and posttraumatic stress disorder. The Journal of clinical psychiatry. 2004;65(10):1314-22.

[6] Tschudin S, Bertea PC, Zemp E. Prevalence and predictors of premenstrual syndrome and premenstrual dysphoric disorder in a population-based sample. Archives of women's mental health. 2010;13(6):485-94

[7] Raja SN, Feehan M, Stanton WR, McGEE R. Prevalence and correlates of the premenstrual syndrome in adolescence.
Journal of the American Academy of Child \& Adolescent Psychiatry. 1992;31(5):783-9.

[8] Bakhshani NM, Mousavi MN, Khodabandeh G. Prevalence and severity of premenstrual symptoms among Iranian female university students. Prevalence. 2009.

[9] Derman O, Kanbur NÖ, Tokur TE, Kutluk T. Premenstrual syndrome and associated symptoms in adolescent girls. European Journal of Obstetrics \& Gynecology and Reproductive Biology. 2004;116(2):201-6.

[10] Rizk DE, Mosallam M, Alyan S, Nagelkerke N. Prevalence and impact of premenstrual syndrome in adolescent schoolgirls in the United Arab Emirates. Acta obstetricia et gynecologica Scandinavica. 2006;85(5):589-98.

[11] Marván ML, Cortés-Iniestra S. Women's beliefs about the prevalence of premenstrual syndrome and biases in recall of premenstrual changes. Health Psychology. 2001;20(4):276.

[12] Drosdzol A, Nowosielski K, Skrzypulec V, Plinta R. Premenstrual disorders in Polish adolescent girls: Prevalence and risk factors. Journal of Obstetrics and Gynaecology Research. 2011;37(9):1216-21.

[13] Tenkir A, Fisseha N, Ayele B. Premenstrual syndrom: prevalence and effect on academic and social performances of students in Jimma University, Ethiopia. Ethiopian Journal of Health Development. 2004;17(3):181-8.

[14] Sadler C, Smith H, Hammond J, Bayly R, Borland S, Panay N, et al. Lifestyle factors, hormonal contraception, and premenstrual symptoms: the United Kingdom Southampton Women's Survey. Journal of Women's Health. 2010;19(3):3916.

[15] Erbil N, Karaca A, Kırıș T. Investigation of premenstrual syndrome and contributing factors among university students. Turk J Med Sci. 2010;40(4):565-73.

[16] Chang AM, Holroyd E, Chau JP. Premenstrual syndrome in employed Chinese women in Hong Kong. Health Care for Women International. 1995;16(6):551-61.

[17] Heinemann LA, Minh TD, Filonenko A, Uhl-Hochgräber K. Explorative evaluation of the impact of severe premenstrual disorders on work absenteeism and productivity. Women's Health Issues. 2010;20(1):58-65.

[18] Tabassum S, Afridi B, Aman Z, Tabassum W, Durrani R. Premenstrual syndrome: frequency and severity in young college girls. JOURNAL-PAKISTAN MEDICAL ASSOCIATION. 2005;55(12):546.

[19] Freeman EW, Sondheimer SJ. Premenstrual dysphoric disorder: recognition and treatment. Primary care companion to the Journal of clinical psychiatry. 2003;5(1):30.

[20] Reid RL, Yen S. Premenstrual syndrome. American Journal of Obstetrics and Gynecology. 1981;139(1):85-104.

[21] Dickerson LM, Mazyck PJ, Hunter MH. Premenstrual syndrome. American family physician. 2003;67(8):1743-52. 